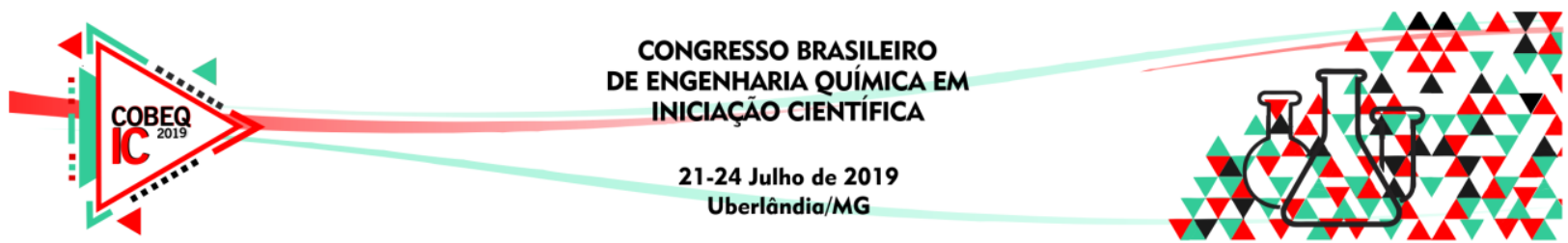

\title{
PRÉ-TRATAMENTO E HIDRÓLISE ENZIMÁTICA DA BIOMASSA LIGNOCELULÓSICA DE MILHO EM REATOR BATELADA
}

\author{
A. P. DRESCH ${ }^{1}$, A. BUENO ${ }^{1}$, J. F. FUHR ${ }^{1}$, G. M. MIBIELLI ${ }^{1}$ e J. P. BENDER ${ }^{1}$ \\ ${ }^{1}$ Universidade Federal da Fronteira Sul, curso de Engenharia Ambiental e Sanitária \\ E-mail para contato: alinepdresch@gmail.com
}

\begin{abstract}
RESUMO - A necessidade de diversificação da matriz energética mundial vem intensificando as pesquisas relacionadas às energias renováveis, como aquelas provenientes de resíduos agroindustriais. Assim, esse estudo objetivou avaliar a etapa de pré-tratamento da biomassa lignocelulósica, visando a obtenção de açúcares. Para tanto, realizou-se um Planejamento Fatorial Completo para avaliar as variáveis "concentração de glicerina" e "tipo de agente químico" na etapa de pré-tratamento, tendo como variável resposta a concentração de açúcares fermentescíveis. A hidrólise enzimática da biomassa tratada foi realizada utilizando-se os complexos enzimáticos $\mathrm{Ctec} 2 \mathrm{e} \mathrm{Htec} 2$, nas porcentagens de $2 \mathrm{e}$ $0,5 \%(\mathrm{v} / \mathrm{m})$, respectivamente. A glicerina apresentou um efeito negativo no processo, enquanto a adição dos agentes químicos hidróxido de cálcio e óxido de cálcio apresentaram resultados positivos na quebra da rigidez estrutural da biomassa, obtendo-se um mosto com concentrações próximas a 0,31 $\mathrm{g}_{\text {açúcares }} / \mathrm{g}_{\text {biomassa }}$ após hidrólise enzimática, o que corresponde a aproximadamente $47,4 \%$ de rendimento em relação a quantidade de celulose e hemicelulose presentes na biomassa. Diante disso, demonstrou-se que a biomassa residual do milho é uma matéria-prima promissora para produção de bioetanol. No que se refere à utilização de agitação mecânica, empregando impelidor do tipo hélice naval, foi possível afirmar que, trabalhando-se com razão sólido/líquido de 60 g/L, o mesmo manteve o meio homogêneo, sendo favorável à produção de bioetanol lignocelulósico em reator tipo batelada.
\end{abstract}

\section{INTRODUÇÃO}

A busca por alternativas renováveis de energia vem crescendo, sendo motivada principalmente pelo aumento do consumo energético e alta dependência dos combustíveis fósseis, que são prejudiciais ao meio ambiente e têm suas fontes escassas e mal distribuídas. Uma das alternativas são os biocombustíveis obtidos a partir de resíduos agroindustriais, que oferecem flexibilidade e menor preço. Além disso, esse tipo de bioetanol pode ser produzido em larga escala sem que seja necessário o aumento da área plantada (Rabelo, 2010).

Para obter-se etanol a partir da biomassa lignocelulósica, é necessária uma etapa de prétratamento, que tem a função de facilitar o acesso dos agentes hidrolíticos ao interior da estrutura, o que aumenta o custo total do processo. Nesse sentido, pesquisas têm estudado a adição de outras substâncias além daquelas já utilizadas, como a glicerina, composto residual 


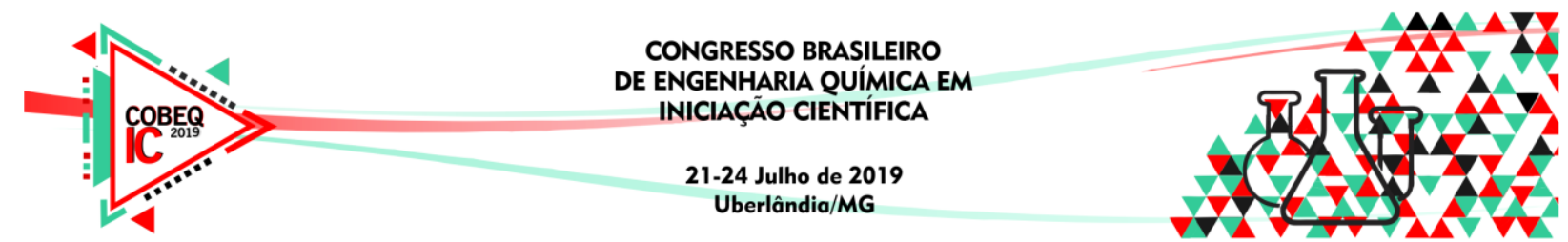

da produção de biodiesel. Por ser um solvente orgânico e ter estrutura polar, a glicerina pode penetrar na estrutura vegetal, contribuindo na quebra da lignina (Martin et al., 2011).

As substâncias surfactantes também têm se destacado. Relatos da literatura indicam que sua adição melhora a conversão da biomassa em açúcares. Segundo Zhang et al. (2017), a presença de surfactantes no meio aumenta a atividade e estabilidade das celulases, intensificando as interações entre essas enzimas e seus substratos.

Outra dificuldade encontrada na produção de bioetanol lignocelulósico é a eficiência do processo de transferência de calor e massa, variável que controla a velocidade da reação e tem grande impacto no custo final. Esse processo é acelerado pela agitação do meio mediante agitadores mecânicos, que impõem movimento ao fluido (Corrêa, 2016).

Tendo, portanto, como desafio atual, o melhoramento das etapas de pré-tratamento e hidrólise enzimática dos resíduos lignocelulósicos, assim como, levando em conta a importância de se estudar ambos os processos em uma unidade experimental voltada ao uso industrial, o presente trabalho teve como objetivo buscar a maximização na obtenção de açúcares fermentescíveis, em reator batelada, avaliando o sistema mecânico de agitação.

\section{METODOLOGIA}

\subsection{Materiais}

Para a execução dos testes de pré-tratamento e hidrólise enzimática, foi adaptada uma unidade experimental, conforme Figura 1.

Figura 1 - Representação esquemática do aparato experimental: (1) motor de agitação; (2) reator; (3) impelidor; (4) chicanas; (5) banho de óleo e; (6) chapa aquecedora.

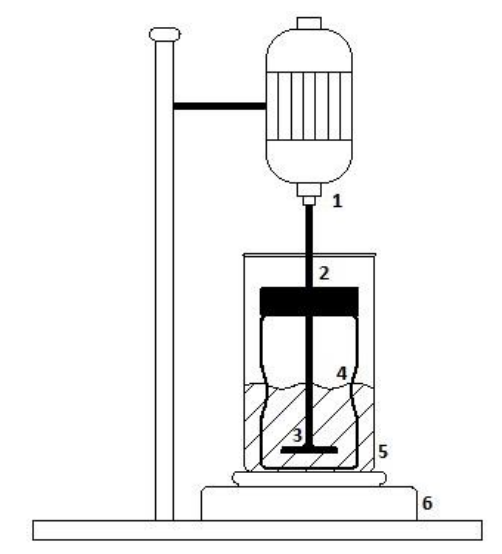

Fonte: próprios autores, 2018.

O agitador mecânico utilizado possui marcação analógica, com velocidade variando de 1 a 8 . Cada um dos pontos corresponde a aproximadamente $225 \mathrm{rpm}$, porém, apenas quando utilizado somente água como fluido a ser agitado.

A biomassa utilizada foi obtida nas áreas experimentais da Universidade Federal da Fronteira Sul - Campus Chapecó, e corresponde ao híbrido do milho DKB 177 Pro 2. A 


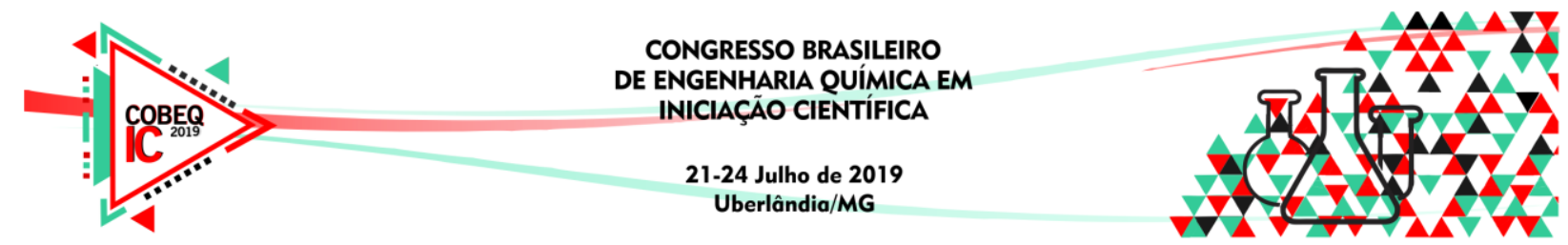

mesma foi seca em estufa a $50^{\circ} \mathrm{C}$, passou por moinho de facas e peneiramento com peneiras de 30 mesh, resultando em partículas menores que $0,6 \mathrm{~mm}$.

\subsection{Procedimentos}

A caracterização da biomassa foi realizada por Bohn et al. (2018). Foram determinados os teores de umidade, cinzas, extrativos, lignina total, celulose e hemicelulose, utilizando a metodologia descrita por Rabelo (2010).

Partindo dos resultados da caracterização, foram realizados testes preliminares para avaliação do sistema reacional e obtenção de um meio homogêneo. Os parâmetros avaliados foram: razão sólido/líquido; tempo de pré-tratamento e; velocidade de agitação.

A partir das condições definidas nos testes preliminares, empregou-se um planejamento experimental fatorial completo $2^{2}$, com triplicata no ponto central, objetivando identificar o efeito de diferentes agentes químicos na etapa de pré-tratamento, que resultasse na maior disponibilização dos constituintes da celulose e hemicelulose para a etapa de hidrólise. Para tanto, investigaram-se os parâmetros "concentração de glicerina" e "tipo de agente químico" utilizado no pré-tratamento. Os tratamentos e níveis investigados estão dispostos na Tabela 1.

Tabela 1 - Matriz do planejamento fatorial completo $2^{2}$, com triplicata no ponto central, empregado para avaliar o efeito de diferentes substâncias adicionadas para pré-tratamento

\begin{tabular}{ccc}
\hline Ensaio & $\begin{array}{c}\text { Concentração de } \\
\text { glicerina (mL) }\end{array}$ & $\begin{array}{c}\text { Óxido de Cálcio/Hidróxido } \\
\text { de Cálcio (g) }\end{array}$ \\
\hline $\mathbf{1}$ & $0,0(-1)$ & $2,4 / 0,0(-1)$ \\
$\mathbf{2}$ & $2,4(+1)$ & $2,4 / 0,0(-1)$ \\
$\mathbf{3}$ & $0,0(-1)$ & $0,0 / 2,4(+1)$ \\
$\mathbf{4}$ & $2,4(+1)$ & $0,0 / 2,4(+1)$ \\
$\mathbf{5}$ & $1,2(0)$ & $1,2 / 1,2(0)$ \\
$\mathbf{6}$ & $1,2(0)$ & $1,2 / 1,2(0)$ \\
$\mathbf{7}$ & $1,2(0)$ & $1,2 / 1,2(0)$ \\
\hline
\end{tabular}

Fonte: próprios autores, 2018.

Para os ensaios, amostras de $12 \mathrm{~g}$ de biomassa foram pesadas e tratadas segundo o estabelecido no planejamento experimental, conforme Tabela 1. Também foi adicionado surfactante Tween 80 na concentração de $2,5 \%$ (v/v de solução). Posteriormente, as amostras foram colocadas sob a influência do agitador mecânico a velocidade 8 e $70^{\circ} \mathrm{C}$, por 12 horas.

Transcorrido o tempo de pré-tratamento, as amostras foram neutralizadas com solução de ácido cítrico $(1 \mathrm{M})$ até $\mathrm{pH}$ ótimo $(5,0)$. As enzimas foram adicionadas nas concentrações de $2 \%$ para Ctec2 e 0,5\% para Htec2 (volume de enzima/m de biomassa), diluídas 1:10 em tampão acetato de sódio $(0,05 \mathrm{~mol} / \mathrm{L}$ e pH 4,8).

As amostras foram novamente colocadas sob agitação mecânica, a $50^{\circ} \mathrm{C}$ e velocidade 8 , por 24 horas. Após o processo, os hidrolisados foram centrifugados; alíquotas foram filtradas em filtros com poros de 0,22 Milipore e diluídas 1:2 em água destilada, sendo analisadas por CLAE (Cromatografia Líquida de Alta Eficiência). 


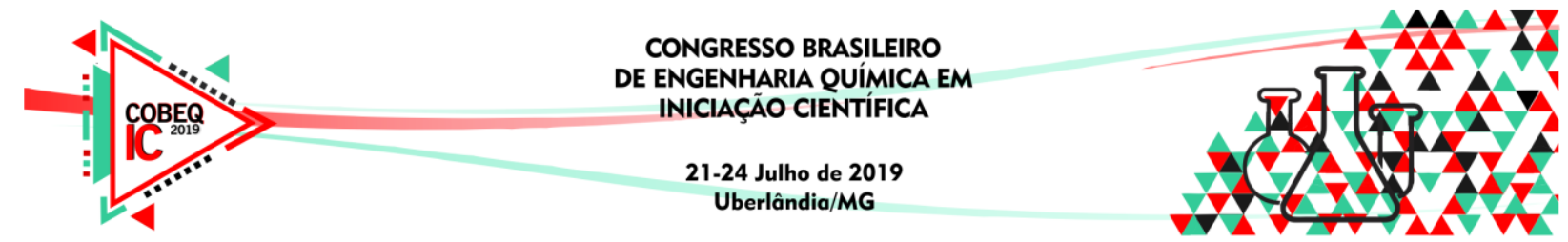

\section{RESULTADOS E DISCUSSÕES}

Conforme indicado por Bohn et al. (2018), a fração caule do milho utilizada neste estudo apresenta, em base seca, $28,44 \%$ de hemicelulose, $37,01 \%$ de celulose, $17,46 \%$ de lignina total, $25,36 \%$ de extrativos e $2,86 \%$ de cinzas. Assim, aproximadamente $65,45 \%$ da biomassa, correspondente aos teores de celulose e hemicelulose, é passível de conversão em açúcares fermentescíveis.

Por meio de testes preliminares foi possível estabelecer condições fixas para as variáveis razão sólido/líquido (60 g/L), tempo de pré-tratamento (12 horas) e velocidade de agitação (velocidade 8 do equipamento), para a obtenção de um meio reacional homogêneo.

Quanto ao planejamento experimental, realizado para avaliar o efeito das variáveis "concentração de glicerina" e "tipo de agente químico", os resultados estão dispostos na Tabela 2. Também é apresentado o valor de rendimento para cada ensaio, calculado com base na concentração de celulose e hemicelulose presente na biomassa $(65,45 \%)$.

Tabela 2 - Rendimento glicosídico obtido com a variação dos agentes de pré-tratamento por meio de planejamento experimental

\begin{tabular}{|c|c|c|c|c|c|c|c|c|}
\hline Ensaio & $\begin{array}{c}\text { Glicerina } \\
(\mathrm{mL} / \mathrm{g})\end{array}$ & $\begin{array}{c}\mathrm{CaO} / \\
\mathrm{Ca}(\mathrm{OH})_{2} \\
(\mathrm{~g} / \mathrm{g})\end{array}$ & $\begin{array}{c}\text { Glicose } \\
(\mathrm{g} / \mathrm{L})\end{array}$ & $\begin{array}{l}\text { Xilose } \\
(\mathrm{g} / \mathrm{L})\end{array}$ & $\begin{array}{c}\text { Celobiose } \\
\text { (g/L) }\end{array}$ & $\begin{array}{c}\mathrm{CT} \\
(\mathrm{g} / \mathrm{L})\end{array}$ & $\underset{\text { biomassa }}{\text { g CT/g }}$ & $\mathbf{R}(\%)^{*}$ \\
\hline 1 & 0 & $2,4 / 0,0(-)$ & $\begin{array}{c}15,97 \pm \\
0,06\end{array}$ & $\begin{array}{c}7,64 \pm \\
0,03\end{array}$ & ND & $\begin{array}{c}23,61 \pm \\
0,09\end{array}$ & $\begin{array}{c}0,39 \pm \\
0,00\end{array}$ & $\begin{array}{c}60,12 \pm \\
0,23\end{array}$ \\
\hline 2 & ) & $2,4 / 0,0(-)$ & $\begin{array}{c}14,72 \pm \\
0,30\end{array}$ & $\begin{array}{c}4,43 \pm \\
0,03\end{array}$ & ND & $\begin{array}{c}19,15 \pm \\
0,31\end{array}$ & $\begin{array}{c}0,32 \pm \\
0,01\end{array}$ & $\begin{array}{c}48,76 \pm \\
0,80\end{array}$ \\
\hline 3 & & $0,0 / 2,4(+)$ & $\begin{array}{c}13,31 \pm \\
1,39\end{array}$ & $\begin{array}{c}6,10 \pm \\
0,49\end{array}$ & ND & $\begin{array}{c}19,41 \pm \\
1,87\end{array}$ & $\begin{array}{c}0,32 \pm \\
0,03\end{array}$ & $\begin{array}{c}49,42 \pm \\
4,77\end{array}$ \\
\hline 4 & & $0,0 / 2,4(+)$ & $\begin{array}{c}12,85 \pm \\
1,10\end{array}$ & $\begin{array}{c}3,83 \pm \\
0,34\end{array}$ & $\begin{array}{c}0,54 \pm \\
0,06\end{array}$ & $\begin{array}{c}17,23 \pm \\
1,49\end{array}$ & $\begin{array}{c}0,29 \pm \\
0,02\end{array}$ & $\begin{array}{c}43,86 \pm \\
3,78\end{array}$ \\
\hline 5 & (0) & $1,2 / 1,2(0)$ & $\begin{array}{c}11,75 \pm \\
0,67\end{array}$ & $\begin{array}{c}3,31 \pm \\
0,21\end{array}$ & $\begin{array}{c}0,69 \pm \\
0,05\end{array}$ & $\begin{array}{c}15,75 \pm \\
0,93\end{array}$ & $\begin{array}{c}0,26 \pm \\
0,02\end{array}$ & $\begin{array}{c}40,12 \pm \\
2,36\end{array}$ \\
\hline 6 & $1,2(0)$ & $1,2 / 1,2(0)$ & $\begin{array}{c}11,77 \pm \\
0,59\end{array}$ & $\begin{array}{c}4,45 \pm \\
0,85\end{array}$ & $\begin{array}{c}0,73 \pm \\
0,01\end{array}$ & $\begin{array}{c}16,94 \pm \\
1,44\end{array}$ & $\begin{array}{c}0,28 \pm \\
0,02\end{array}$ & $\begin{array}{c}43,14 \pm \\
3,66\end{array}$ \\
\hline 7 & $1,2(0)$ & $1,2 / 1,2(0)$ & $\begin{array}{c}11,76 \pm \\
0,01\end{array}$ & $\begin{array}{c}3,88 \pm \\
0,80\end{array}$ & $\begin{array}{c}0,71 \pm \\
0,03\end{array}$ & $\begin{array}{c}16,35 \pm \\
0,84\end{array}$ & $\begin{array}{c}0,27 \pm \\
0,01\end{array}$ & $\begin{array}{c}41,63 \pm \\
2,14\end{array}$ \\
\hline
\end{tabular}

(ND): Não detectado; (CT): Carboidratos totais; $\mathrm{R}\left({ }^{*}\right)$ : Rendimento percentual de celulose e hemicelulose.

Fonte: próprios autores, 2018.

Analisando os resultados da Tabela 2, observa-se que a melhor condição encontrada foi no ensaio 1 , que obteve $0,39 \mathrm{~g}$ de açúcares por grama de biomassa, correspondendo a um rendimento de $60,12 \%$.

A partir da análise estatística realizada, foi possível perceber que as duas variáveis estudadas apresentaram efeito significativo negativo. Isso revela que, no caso da glicerina, quanto menor for a concentração adicionada, melhor a conversão. Para o parâmetro agente 


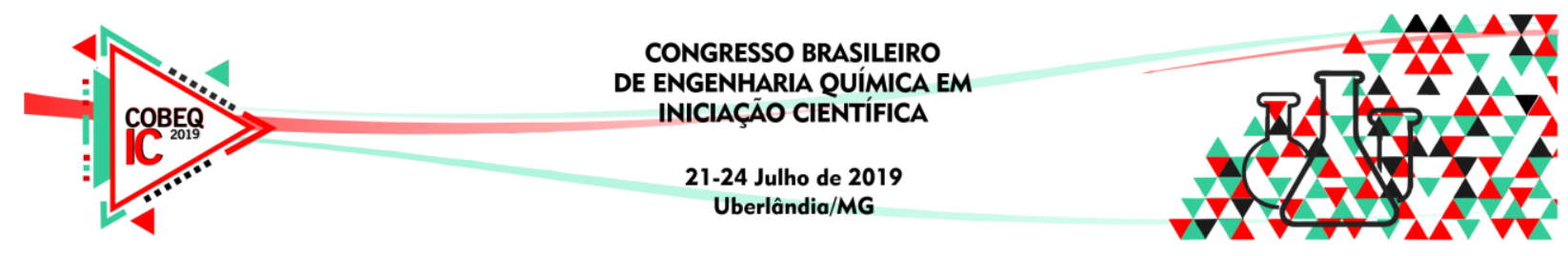

químico, o tratamento negativo representa o óxido de cálcio, o que significa que esse composto apresenta melhores resultados em termos de rendimento glicosídico frente à utilização do hidróxido de cálcio. O efeito da interação das variáveis é não significativo.

A partir dos resultados encontrados, tendo como melhor condição o pré-tratamento sem adição de glicerina e com a utilização do óxido de cálcio, realizou-se uma triplicata de ensaios com as mesmas condições, visando repetir o resultado do ensaio 1 ( $0,39 \mathrm{~g} \mathrm{CT/g}$ biomassa).

Porém, no novo teste não foi possível repetir o resultado do ensaio 1 do planejamento. Assim, comparando a média dos quatro ensaios realizados com óxido de cálcio (planejamento experimental e testes de repetição), e seus intervalos de confiança $(\alpha=0,05)$, com os resultados do ensaio 3 do planejamento (realizado com hidróxido de cálcio), é possível observar que, estatisticamente, não existe variação significativa na utilização dos dois compostos, já que há sobreposição dos valores. Essa comparação pode ser observada na Figura 3.

Figura 3 - Comparação entre os resultados de rendimento glicosídico para os ensaios com utilização de óxido de cálcio e hidróxido de cálcio

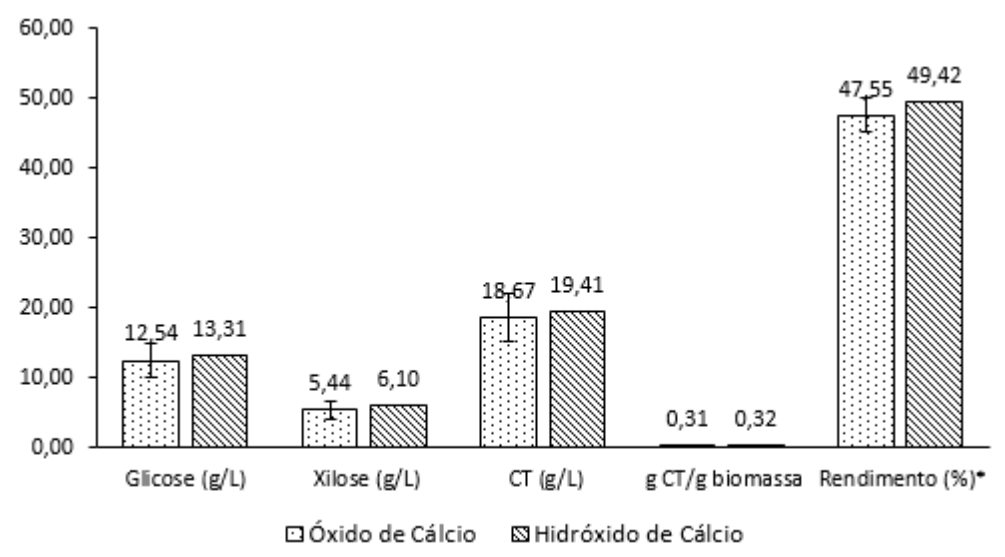

(CT): Carboidratos totais; $(*)$ : Percentual de celulose e hemicelulose convertido.

Fonte: próprios autores, 2018.

O mesmo pode ser concluído se forem comparados o valor médio de rendimento do prétratamento utilizando óxido de cálcio ( $0,31 \mathrm{~g} \mathrm{CT} / \mathrm{g}$ biomassa), com o valor médio obtido nos testes preliminares com hidróxido de cálcio, que resultaram em 0,34 g CT/g biomassa.

Ainda que o melhor resultado do planejamento não tenha se repetido, a média de rendimento dos quatro testes realizados com o óxido de cálcio $(0,31 \mathrm{~g} \mathrm{CT} / \mathrm{g}$ biomassa) pode ser considerada positiva, assim como o resultado de $0,32 \mathrm{~g} \mathrm{CT} / \mathrm{g}$ biomassa obtido pelo hidróxido de cálcio no planejamento experimental, pois estão próximos dos valores encontrados na literatura.

Rabelo (2010), que estudou as etapas de pré-tratamento e hidrólise enzimática utilizando bagaço de cana-de-açúcar, ambos os processos realizados com agitação orbital em shaker, chegou a um valor final de $0,35 \mathrm{~g} \mathrm{CT} / \mathrm{g}$ biomassa, muito próximo ao relatado no presente estudo, frente a nova configuração do sistema. 


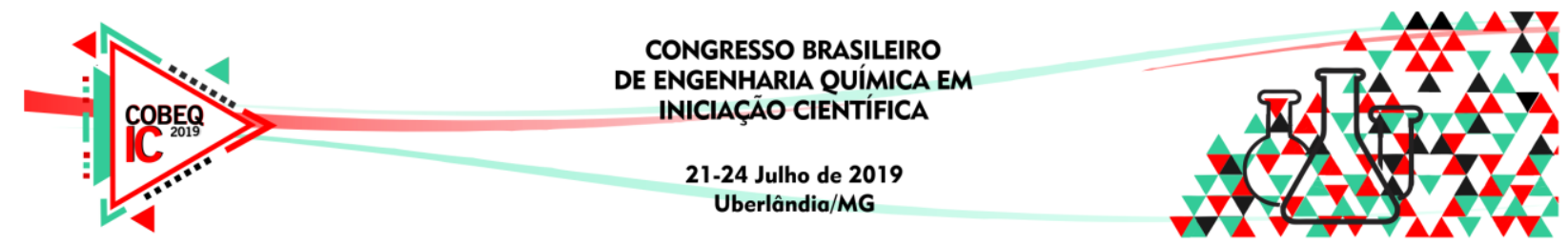

Também, vale destacar que o aparato experimental utilizado para realização dos ensaios não foi dimensionado levando em consideração as características do processo, mas adaptado segundo os equipamentos disponíveis. A escolha dos impelidores, por exemplo, segundo Corrêa (2016), deve levar em conta o tipo de mistura, a capacidade do reator e seu formato, além das propriedades do fluido, principalmente sua viscosidade.

\section{CONCLUSÕES}

Através dos resultados apresentados, foi possível observar que a fração de biomassa residual de milho utilizada no presente trabalho é matéria-prima promissora para produção de bioetanol, apresentando percentual de $65,45 \%$ de celulose e hemicelulose.

No planejamento experimental, realizado para definição do melhor composto prétratante, concluiu-se que ambos os agentes químicos hidróxido de cálcio e óxido de cálcio são eficientes na quebra da rigidez estrutural da biomassa, resultando em valores de 0,32 e $0,31 \mathrm{~g}$ CT/g biomassa após hidrólise enzimática, respectivamente.

Por fim, é possível afirmar que o sistema mecânico de agitação é conveniente aos processos de pré-tratamento e hidrólise enzimática, não resultando em alterações nas estruturas da biomassa e das enzimas, desnaturação dos complexos enzimáticos na etapa de hidrólise ou aceleração dos processos de degradação dos carboidratos, sendo favorável ao avanço das pesquisas relacionadas a produção de etanol lignocelulósico em larga escala.

\section{REFERÊNCIAS}

BOHN, L. R.; DRESCH, A. P.; ALVES, S. L.; MIBIELLI, G. M.; BENDER, J. P. Obtenção de açúcares fermentescíveis a partir da biomassa lignocelulósica de milho para produção de bioetanol. Anais da VIII JIC, Realeza, v. 8, n. 1, 2018.

CORRÊA, L. J. ; BADINO, A. C.; CRUZ, A. J. G. Mixing design for enzymatic hydrolysis of sugarcane bagasse: methodology for selection of impeller configuration. Bioprocess and Biosystems Engineering. [s.1.], v. 39, p. 285-294, 2016.

MARTIN, C; PULS, J.; SAAKE, B; SCHREIBER, A. Effect of glycerol pretreatment on component recovery and enzymatic hydrolysis of sugarcane bagasse. Cellulose Chemistry and Technology. [s.1.], v. 45, p. 487-494, 2011.

RABELO, S. C. Avaliação e otimização de pré-tratamentos e hidrólise enzimática do bagaço de cana-de-açúcar para a produção de etanol de segunda geração. 2010. 447 f. Tese (Doutorado em Engenharia Química). Universidade Estadual de Campinas. Campinas SP, 2010.

ZHANG, H.; YE, G., WEI, Y.; LI, X.; ZHANG, A., XIE, J.. Enhanced enzymatic hydrolysis of sugarcane bagasse with ferric chloride pretreatment and surfactant. Bioresource Technology. [s.1.], v. 229, p. 96-103, abr. 2017. 